\title{
Bildung für eine Nachhaltige Entwicklung im Vergleich mit Globalem Lernen und Umweltbildung
}

\section{Christine Künzli David, Franziska Bertschy und Antonietta Di Giulio}

Derzeit finden sich zahlreiche Initiativen, um Bildung für eine Nachhaltige Entwicklung (BNE) im Bildungssystem zu verankern. Eine zentrale Frage in diesem Zusammenhang stellt sich nach dem Verhältnis zwischen BNE, globalem Lernen und Umweltbildung. Zum einen wird befürchtet, mit BNE komme zum grossen Katalog bisheriger "Bindestrich-Erziehungen“ eine weitere hinzu, zum anderen ist $z u$ beobachten, dass fächerübergreifende Bildungsanliegen und insbesondere globales Lernen und Umweltbildung auffallend oft auf BNE bezogen werden - BNE scheint also offenbar an bereits bestehende fächerübergreifende Bildungsanliegen anschlussfähig.

Der vorliegende Beitrag ist dieser Frage nach dem Verhältnis von BNE und dem Globalen Lernen sowie der Umweltbildung gewidmet.

\section{Einleitung}

Dem Bildungssektor wird eine grosse Bedeutung beigemessen, wenn es darum geht, eine Nachhaltige Entwicklung umzusetzen. Aus diesem Grund wurden von den Vereinten Nationen die Jahre 2005 bis 2014 als Dekade «Bildung für Nachhaltige Entwicklung» (BNE) ausgerufen. Ziel der Dekade ist es u.a., BNE weltweit in den Curricula der verschiedenen Bildungsstufen zu verankern. Derzeit finden sich denn auch zahlreiche Initiativen, um BNE im Bildungssystem umzusetzen. Solche Aktivitäten lassen sich auch für die Schweiz feststellen - insbesondere im Rahmen der Entwicklung der neuen sprachregionalen Lehrpläne, innerhalb derer BNE integriert werden soll. Eine der zentralen Fragen in diesem Zusammenhang ist diejenige nach dem Verhältnis zwischen $\mathrm{BNE}^{1}$ und anderen fächerüberübergreifenden Bildungsanliegen. Dabei werden vor allem zwei Aspekte diskutiert:

Zum einen wird befürchtet, mit BNE komme zum grossen Katalog bisheriger «Bindestrich-Erziehungen» (Schorch, 2007) eine weitere dazu, was den bereits mehrfach kritisierten "gesellschaftlichen Reparaturauftrag», der der Schule 
zugewiesen werde, weiter verschärfe (Apel, 1995). Die Schule werde mit verschiedensten Aufgaben überfrachtet, weil aus gesellschaftlichen Problemfeldern immer neue Erziehungsaufträge resultieren würden: Umwelt-, Konsum-, Friedens-, Bewegungs-, Ernährungs-, Medien-, Dritte-Welt- etc. Erziehung (Einsiedler, 1994). Solche «Bindestrich-Erziehungen» würden, auch wenn sie jeweils für sich berechtigt und sinnvoll erschienen, in ihrer Summe die Volksschule überfordern und den Blick auf die Kernaufgaben von Bildung verstellen. Zum anderen ist im Diskurs zu beobachten, dass gerade solche «Bindestrich-Erziehungen» auffallend oft auf BNE bezogen werden. BNE scheint demzufolge an viele der bestehenden fächerübergreifenden Bildungsanliegen anschlussfähig zu sein (vgl. z.B. Scheunpflug 2007). Nagel, Kern und Schwarz (2006) z.B. bezeichnen verschiedene fächerübergreifende Bildungsanliegen (Gesundheitsbildung, Globales Lernen und Umweltbildung) als "Themenfelder» der BNE. Diese konstatierte Anschlussfähigkeit wiederum hat zur Folge, dass die Frage aufgeworfen wird, was denn das «Neue» von BNE sei, und Befürchtungen laut werden, BNE werde zu einem Auffangbecken für jede Art von Bildungsanliegen (de Haan, 2001). Insgesamt wird BNE aus diesen Gründen oft als diffus (de Haan, 2008; Helbling \& Schwarz, 2005) und schwer zu fassen (Kyburz-Graber, Högger \& Wyrsch, 2000) wahrgenommen. De Haan (2002, S. 14) stellt beispielsweise fest, dass "Abgrenzungen zur Umweltbildung (ist die Beschäftigung mit dem Lebensraum 'Bach', Teil der Umweltbildung oder Teil einer BNE?) (...) ebenso schwierig [seien] wie zur entwicklungspolitischen Bildung (ist die Beschäftigung mit den 'Bildungschancen' von Mädchen in den Sahelstaaten, der entwicklungspolitischen Bildung oder der BNE zuzurechnen?) und zur Friedenspädagogik (ist das Thema 'Nationalsozialismus als Ursache von militärischen Konflikten', der Friedenspädagogik oder der sozialen Seite der Nachhaltigkeit zuzurechnen?)». Von diversen Autorinnen und Autoren wird zudem betont, in BNE gingen verschiedene Themenfelder und Ansätze speziell aus der Umweltbildung und dem Globalen Lernen auf (Bolscho, Hauenschild \& Rode, 2008; Rost, 2002).

Der vorliegende Beitrag stellt die Frage nach dem Verhältnis von BNE und anderen fächerübergreifenden Bildungsanliegen (insbesondere Globales Lernen und Umweltbildung) in den Vordergrund: Inwiefern sind die didaktischen Strukturelemente einer BNE kompatibel mit denjenigen des Globalen Lernens und der Umweltbildung und inwiefern kann BNE somit als Fortführung dieser fächerübergreifenden Bildungsanliegen verstanden werden resp. diese fächerübergreifenden Bildungsanliegen umfassen?

Fächerübergreifende Bildungsanliegen sind Bildungsaufträge an die Schule, welche sich nicht in einem der Fachbereiche verorten lassen, sondern mit einer eigenen Zielsetzung an die Schule herantreten. Das jeweilige Anliegen wird entweder als allgemeines Prinzip von Schule und Unterricht verstanden oder innerhalb eines multiperspektivischen Faches (wie z.B. dem Sachunterricht) resp. fächerübergreifend mit Bezug zu mehreren Fächern verankert. Die wissen- 
schaftliche Beschäftigung mit diesen Anliegen, die ihren eigentlichen Ursprung, wenn auch in unterschiedlichem Mass, in der Reaktion auf gesellschaftliche Anliegen und Problemlagen haben, geschieht in entsprechenden Teildisziplinen der Erziehungswissenschaft (Herzog \& Künzli David, 2007). Fächerübergreifende Bildungsanliegen stammen demnach ursprünglich nicht aus einem wissenschaftlichen bzw. bildungstheoretischen Diskurs (Scheunpflug, 2003). In der Auseinandersetzung mit solchen Bildungsanliegen vermischen sich aus den genannten Gründen oftmals auch unterschiedliche pädagogische Diskurse - so z.B. bildungstheoretische, bildungspolitische sowie der Diskurs in pädagogischen Professionen (Herzog \& Künzli David, 2007). Aus diesem Grund werden auch in diesem Artikel verschiedene Diskussionsstränge aufgenommen.

Im Artikel werden Unterschiede und Gemeinsamkeiten zwischen fächerübergreifenden Bildungsanliegen - Umweltbildung und Globales Lernen - und BNE herausgearbeitet, um auf dieser Grundlage Möglichkeiten der Integration von fächerübergreifenden Bildungsanliegen in das Bildungssystem zu diskutieren.

Dazu werden Ergebnisse aus einem inhaltsanalytischen Vergleich (Mayring, 2000) präsentiert. Aufhänger für die Darstellung der Ergebnisse sind Aussagen zu BNE, die im Zusammenhang mit der Frage nach Identität, Anschlussfähigkeit und Differenz zu anderen fächerübergreifenden Bildungsanliegen immer wieder zu hören sind.

Der Vergleich stützt sich auf umfassende didaktische Konzepte der BNE, der Umweltbildung und des Globalen Lernens. Didaktische Konzepte beinhalten theoretisch konsistente, systematische Aussagen zu verschiedenen didaktischen Strukturelementen, wobei Lernziele, didaktische Prinzipien, Unterrichtsinhalte und die Legitimationsgrundlagen ${ }^{2}$ die wichtigsten solchen Strukturelemente sind und im vorgenommenen Vergleich systematisch untersucht wurden. Insgesamt versuchen didaktische Konzepte wissenschaftlich hergeleitete Antworten auf die Fragen zu geben, die mit der Planung, Durchführung und Reflexion von Bildungsveranstaltungen auftreten (Hoppe, 1996). In den Vergleich wurden aus diesem Grunde nur Arbeiten einbezogen, die sich in Form eines didaktischen Konzepts zum entsprechenden Bildungsanliegen äussern, und nicht solche, in denen z.B. Aspekte des Bildungsanliegens für empirische Arbeiten operationalisiert wurden oder Umsetzungsbeispiele in Bezug auf den Unterricht aufgezeigt werden. ${ }^{3}$

Für jedes der zu vergleichenden Bildungsanliegen wurde ein deutschsprachiges $^{4}$ didaktisches Konzept (inkl. der dazugehörigen Legitimationsgrundlage) analysiert. ${ }^{5}$ Für "Globales Lernen" ist dies das didaktische Konzept von Scheunpflug und Schröck (2000), für «Umweltbildung» das von Kyburz-Graber et al. $\left(1997,2000^{6}\right)$, und für BNE das von Künzli David und Bertschy $(2008)^{7}$. 


\section{Didaktisches Konzept zu BNE}

Das im Vergleich verwendete Konzept zu BNE wird nachfolgend dargelegt. Im Vergleich der didaktischen Konzepte bildet dieses Konzept die Vergleichsgrundlage und wird aus diesem Grunde etwas ausführlicher dargestellt.

\section{Legitimationsgrundlagen}

Grundlegend für dieses Konzept zu BNE ist das Nachhaltigkeitsverständnis der Vereinten Nationen (Di Giulio, 2004), wonach eine Entwicklung angestrebt wird, "die die Bedürfnisse der Gegenwart befriedigt, ohne zu riskieren, dass zukünftige Generationen ihre eigenen Bedürfnisse nicht befriedigen können» (Hauff, 1987, S. 46). Nachhaltige Entwicklung stellt eine regulative Idee dar, d.h. sie ist eine Orientierungsgrösse für menschliche Such- und Lernprozesse, aus der sich nicht ein für allemal konkrete Handlungsanweisungen ableiten lassen (vgl. z.B. Kübler, Kissling-Näf \& Zimmermann, 2001; Minsch, Eberle, Meier \& Schneidewind, 1996).

BNE muss - wie jeder Teil des allgemeinbildenden Bildungssystems - einen Beitrag zu übergeordneten Bildungszielen leisten. Durch Bildung sollen sich Menschen als eigenständige Personen in der Welt zurechtfinden, Verantwortung übernehmen können und über ein kritisches Bewusstsein ohne Verlust eines bejahenden Lebensgefühls sowie über Handlungsbereitschaften verfügen (Durdel, 2002). Eine Instrumentalisierung der heranwachsenden Generation für gegenwärtige politische Ziele ist mit der Idee von Bildung nicht vereinbar und muss ausgeschlossen werden können. Damit kann auch die politische Idee der Nachhaltigkeit nicht tel quel als pädagogischer Auftrag erscheinen, vielmehr muss dieser aufgrund pädagogischer Überlegungen formuliert werden (Herzog \& Künzli David, 2007). Die Legitimationsgrundlage von BNE kann somit nur die regulative Idee der Nachhaltigen Entwicklung sein, nicht deren kontextgebundene Konkretisierung.

\section{Didaktische Strukturelemente}

Aus der Leitidee einer Nachhaltigen Entwicklung und orientiert an einem modernen Bildungsverständnis ergeben sich die nachstehend ausgeführten übergeordneten Lernziele, didaktischen Prinzipien und Kriterien zur Inhaltsauswahl (Bertschy, Gingins, Künzli, Di Giulio \& Kaufmann-Hayoz, 2007; Künzli David, 2007).

\section{Lernziele}

Als übergeordnetes Leitziel lässt sich folgendes formulieren: Die Schüler und Schülerinnen haben die Bereitschaft und die Fähigkeit, sich an gesellschaftlichen Aushandlungs- und Mitgestaltungsprozessen in Bezug auf eine Nachhaltige Entwicklung zu beteiligen. Sie besitzen ein Bewusstsein für die Bedeutung einer 
Nachhaltigen Entwicklung und die Einsicht in die Mitverantwortlichkeit aller in Bezug auf soziokulturelle, ökonomische und ökologische Entwicklungen sowie auf deren Zusammenwirken. ${ }^{8}$ Dieses Leitziel stellt eine allgemeine Zielorientierung dar und lässt sich in Form von Richtzielen, die zu erwerbende Kompetenzen umschreiben, genauer bestimmen. ${ }^{9}$

Kompetenzbereich selbstständig handeln

Die Schülerinnen und Schüler können

a) die Idee der Nachhaltigkeit als wünschbares Ziel der gesellschaftlichen Entwicklung sowie alternative Auffassungen der Gesellschaftsentwicklung kritisch beurteilen.

b) eigene und fremde Visionen aber auch gegenwärtige Entwicklungstrends im Hinblick auf eine Nachhaltige Entwicklung beurteilen.

c) unter den Bedingungen von Unsicherheit, Widerspruch und unvollständigem Wissen begründete Entscheidungen, die den Anforderungen einer Nachhaltigen Entwicklung genügen, treffen.

d) persönliche, gemeinsame und delegierte Kontrollbereiche in Bezug auf eine Nachhaltige Entwicklung realistisch einschätzen und nutzen.

Kompetenzbereich Instrumente und Medien interaktiv nutzen

e) Die Schülerinnen und Schüler sind in der Lage, sich im Bereich Nachhaltiger Entwicklung zielgerichtet $\mathrm{zu}$ informieren und die Informationen für Entscheidungen im Sinne einer Nachhaltigen Entwicklung effizient einzusetzen.

Kompetenzbereich Handeln in sozial heterogenen Gruppen

Die Schülerinnen und Schüler

f) können mit anderen Menschen zusammen Visionen in Bezug auf eine Nachhaltige Entwicklung erarbeiten und Schritte zur Umsetzung konzipieren und veranlassen.

g) sind in der Lage, Entscheidungen hinsichtlich Nachhaltiger Entwicklung gemeinsam mit anderen auszuhandeln.

\section{Didaktische Prinzipien}

Es wird zwischen allgemeinen und spezifischen didaktischen Prinzipien unterschieden, wobei allgemeine didaktische Prinzipien solche sind, die in vielen anderen Fachbereichen und Bildungsanliegen ebenfalls eine wichtige Rolle spielen und somit nicht spezifisch für BNE sind. Die einzelnen didaktischen Prinzipien sind für die Umsetzung von BNE je notwendig, jedoch noch nicht hinreichend. Die Umsetzung von BNE gemäss dem hier vorgestellten Konzept bedingt, dass in einer Unterrichtseinheit alle didaktischen Prinzipien im Hinblick auf die übergeordneten Richtziele handlungsleitend sind.

Aus Platzgründen werden nachfolgend nur die spezifischen didaktischen Prinzipien erläutert. 
Visionsorientierung: Unterrichtsplanung und -durchführung sollen sich am Entwurf einer erwünschten Zukunft, an einer Vision, orientieren. Auf diese Weise wird ein positiver, optimistischer Zugang zu gesellschaftlichen Entwicklungen ermöglicht; im Zentrum stehen nicht gesellschaftliche Probleme oder Katastrophenszenarien. Dies bedeutet jedoch nicht, dass gesellschaftlich relevante Probleme nicht angesprochen werden sollen - im Gegenteil. Die Thematisierung von Problemen hat jedoch einen anderen Hintergrund und Zweck: Die Schüler und Schülerinnen setzen sich themenspezifisch mit Zukunftsentwürfen sowie mit Möglichkeiten, Bedingungen und Einschränkungen der Realisierung solcher Visionen auseinander. Hierzu sind einerseits ein Verstehen der gegenwärtigen (auch problematischen) gesellschaftlichen Wirklichkeiten und deren historisches Gewordensein, aber andererseits auch kreatives und konstruktives Denken essenziell. In diesem Sinne stehen nicht Probleme und ihre Lösungen, sondern die Visionen und ihre Erreichbarkeit im Vordergrund.

Vernetzendes Lernen: Das didaktische Prinzip des vernetzenden Lernens umfasst grundsätzlich zwei Aspekte: Erstens müssen im Unterricht verschiedene Perspektiven (Akteur- und Fachperspektiven) zu einem Unterrichtsgegenstand aufgezeigt werden. Zweitens müssen diese Perspektiven explizit und angeleitet im Hinblick auf den Gegenstand bzw. auf eine übergeordnete Fragestellung hin verknüpft werden. Das Unterrichtsgeschehen soll die Lernenden also zu Vernetzungen hinführen und ihnen dabei Hilfestellungen bieten. Es darf nicht stillschweigend davon ausgegangen werden, dass die Lernenden von selbst Wissen aus verschiedenen Fachbereichen oder Sichtweisen unterschiedlicher Akteure miteinander in eine Beziehung bringen oder bei konkreten Entscheidungen beiziehen können (Bertschy et al., 2007; Fien, 2001).

Thematisch muss der Unterricht Vernetzungen in verschiedenen Bereichen ermöglichen. So soll er

- Zusammenhänge zwischen lokalen und globalen Gegebenheiten und Ereignissen sowie gesamtgesellschaftliche Auswirkungen,

- Zusammenhänge zwischen heutigen Geschehnissen und Entscheidungen und zukünftige Generationen und

- Zusammenhänge zwischen der ökologischen, ökonomischen und soziokulturellen Dimension aufzeigen.

Partizipationsorientierung: Partizipationsorientierung als didaktisches Prinzip bezieht sich auf zwei Ebenen: Zum einen auf die Klasse als Gesamtheit, die bei Belangen, die für alle Schülerinnen und Schüler von Relevanz sind, einbezogen werden soll, zum anderen auf das einzelne Kind, das in Bereichen, die es persönlich betreffen, (mit)entscheiden soll. Der Grad der Partizipation kann je nach Kontext und Situation unterschiedlich sein. Dabei ist es zentral, dass die Partizipationsorientierung nicht auf Aspekte sozialen Lernens reduziert wird. Partizipationsorientierung muss vielmehr die Auseinandersetzung mit dem gesellschaftlichen Eingebundensein, mit Fragen nach Machtverhältnissen, Herrschaft, 
gesellschaftlicher Organisation von Interessen und mit der Reflexion von Kontrollmöglichkeiten im Hinblick auf eine Nachhaltige Entwicklung umfassen.

\section{Unterrichtsinhalte}

Aus der Idee der Nachhaltigkeit lässt sich kein verbindlicher Inhaltskanon herleiten; es ist an vielen Gegenständen möglich, über Nachhaltige Entwicklung zu reflektieren und zu lernen, gesellschaftliche Prozesse im Hinblick auf eine Nachhaltige Entwicklung mitzugestalten. Dies wiederum bedeutet nicht, dass die Auswahl und Ausrichtung der Unterrichtsgegenstände beliebig ist und alle gleichermassen geeignet wären. Entscheidend sind folgende Inhaltsauswahl- und -ausrichtungskriterien:

Die Gegenstände müssen sich eignen, um

- Zusammenhänge zwischen lokalem und globalem Geschehen aufzuzeigen;

- Veränderungen über längere Zeiträume zu thematisieren;

- Vernetzungen zwischen der soziokulturellen, ökologischen und ökonomischen Dimension, die Interessenlagen und Wertvorstellungen verschiedener Akteure sowie gesamtgesellschaftliche Interessen erkennbar zu machen.

In der Ausrichtung des Unterrichts

- müssen die Haupt- und Nebenfolgen von Entscheidungen oder Handlungen thematisiert werden;

- müssen Bezüge zur Lebenswelt der Lernenden hergestellt werden;

- muss gewährleistet sein, dass an wenigen ausgewählten Beispielen grundlegende Einsichten gewonnen werden können (Exemplarität und Transfer).

\section{Umweltbildung, Globales Lernen und BNE im Vergleich}

Nachfolgend werden ausgewählte Ergebnisse aus dem Vergleich präsentiert. Aus Platzgründen ist es nicht möglich, die analysierten didaktischen Konzepte zu Umweltbildung und zu Globalem Lernen umfassend darzulegen - es werden lediglich die didaktischen Strukturelemente vorgestellt, auf die sich der Vergleich bezieht.

Vernetzungen im Hinblick auf ökologische und soziokulturelle Aspekte sind nicht nur bei BNE wichtig

Mit diesem Argument sind aus didaktischer Sicht die didaktischen Prinzipien und die Inhaltsauswahl angesprochen. Das Argument wird in besonderem Masse bezogen auf den Vergleich Umweltbildung - BNE vorgebracht. Deshalb soll nun exemplarisch anhand des didaktischen Konzepts der sozio-ökologischen Umweltbildung (Kyburz-Graber et al., 1997, 2000) ${ }^{10}$ diskutiert werden, ob und inwiefern sich Umweltbildung und BNE in Bezug auf die Frage der Inhaltsauswahl sowie der didaktischen Prinzipien unterscheiden. 
Den beiden didaktischen Strukturelementen "didaktische Prinzipien» sowie «Inhaltsauswahlkriterien» entsprechen am ehesten diejenigen Ausführungen, die Kyburz-Graber et al. (2000) unter den Stichworten «Kriterien» und «didaktische Komponenten» behandeln. Aus diesem Grunde werden diese für den Vergleich herangezogen:

Als übergeordnetes Auswahlkriterium für Inhalte wird im Konzept ausgeführt: «Unterrichtsthemen von sozio-ökologischer Umweltbildung sind Umweltprobleme und nachhaltige Entwicklung in konkreten Handlungssystemen» (Kyburz-Graber et al., 2000, S. 20).

Als Kriterien werden angegeben:

- «Nachhaltige Entwicklung ist kein eigenständiges Unterrichtsthema.

- Der Gegenstand enthält ökologische, ökonomische und sozial-gesellschaftliche Aspekte, die in einem kontroversen und komplexen Verhältnis zueinander stehen.

- Die Problemlösung auf Rezepte und individuelle Verhaltensweisen zu reduzieren, wird dem Leitbild einer Nachhaltigen Entwicklung nicht gerecht.

- Ein Verständnis für das Leitbild der nachhaltigen Entwicklung und dessen Bedeutung entwickelt sich in einem reflexiven Lernprozess» (ebd., S. 45).

In Bezug auf das zweite Kriterium wird betont, dass die ökologischen, ökonomischen und sozial-gesellschaftlichen Aspekte nicht einfach additiv nebeneinander gestellt werden dürften. Vielmehr soll in der Bearbeitung zum Ausdruck kommen, dass sie funktional zusammenhängen.

Die drei didaktischen Komponenten sind folgende: «Problemorientierung [Hervorhebung durch die Autorinnen] meint die Wahl und Bearbeitung von Themen, welche sich erstens durch Komplexität, zweitens durch Zielkonflikte und drittens durch ein ungelöstes Problem auszeichnen» (ebd., S. 45). Erfahrungsbezug bedeutet, Erfahrungen - sowohl vorhandene als auch im Lernprozess gemachte bewusst und kommunizierbar zu machen und sie zu reflektieren. Dies ermöglicht auch die Erkenntnis, "dass ein und derselbe Sachverhalt aus unterschiedlichen Perspektiven unter Umständen ganz unterschiedlich interpretiert und beurteilt wird» (ebd., S. 47). Kooperation beschreibt, wie Lehrende und Lernende in einem gemeinsam gestalteten Unterrichtsvor-haben zusammenarbeiten und Aufgabenbereiche abgrenzen. Da jedes Individuum ein Problem nur teilweise erfassen kann und eine spezifische Perspektive darauf hat, soll durch die lernende Kooperation zu angemesseneren Lösungen gekommen werden. Dies hilft den Lernenden, «den bearbeiteten Gegenstand (besser) zu verstehen» (ebd., S. 61). Gleichzeitig hat die lernende Kooperation «eine qualifizierende Funktion über das inhaltliche Lernen hinaus» (ebd., S. 59). So werden die Schülerinnen und Schüler dadurch z.B. «für gemeinschaftliche Urteilsfähigkeit in Gruppen qualifiziert» (ebd., S. 59). 


\section{Ergebnis des Vergleichs}

Beim Vergleich dieses Konzepts mit dem zu BNE im Hinblick auf didaktische Prinzipien und Kriterien der Inhaltsauswahl finden sich sowohl Gemeinsamkeiten als auch Differenzen bis hin zu Unvereinbarkeiten:

Gemeinsamkeiten und Überschneidungen finden sich bei bestimmten didaktischen Prinzipien (z.B. in Bezug auf Handlungs- und Reflexionsorientierung, Zugänglichkeit sowie entdeckendes Lernen), bestimmten Inhaltsauswahlkriterien sowie im Grundverständnis von Nachhaltiger Entwicklung. Gemeinsamkeiten finden sich zudem in der Bedeutung, die beide Konzepte der Partizipationsorientierung zukommen lassen. In den Ausführungen zu vernetzendem Lernen sowie zu den Inhaltsauswahlkriterien lassen sich Differenzen ausmachen: Im Konzept zur Umweltbildung spielen im Gegensatz zu dem zu BNE folgende Vernetzungsbereiche keine zentrale Rolle: Verbindung von Gegenwart und Zukunft, Verbindung von lokaler mit globaler Perspektive. Im Vergleich der beiden Konzepte gibt es aber auch Widersprüche bzw. Unvereinbarkeiten: (1) Das Umweltbildungs-Konzept geht thematisch von Umweltanliegen aus, während bei BNE sowohl ökologische als auch soziokulturelle und/oder wirtschaftliche Themen den Ausgangspunkt bilden können. (2) Im Umweltbildungs-Konzept steht die Problemorientierung im Zentrum, bei dem zu BNE die Visionsorientierung, was sich bis zu einem gewissen Grad ausschliesst (auch wenn gemäss letzterem Probleme durchaus behandelt werden).

\section{Die Befähigung zum Umgang mit den Auswirkungen der Globalisierung ist in verschiedenen Bildungsanliegen zentral}

Dieses Argument zielt auf das didaktische Strukturelement der Lernziele. Da es oftmals beim Vergleich BNE - Globales Lernen vorgebracht wird, soll es exemplarisch am didaktischen Konzept von Scheunpflug und Schröck (2000) zu Globalem Lernen geprüft werden. Dabei werden alle Ausführungen aus dem Konzept zu Globalem Lernen beigezogen, die sich zu Lernzielen äussern.

Das Globale Lernen zielt in erster Linie auf den Umgang mit der Globalisierung ab, d.h. auf das Wissen um die Entwicklung zur Weltgesellschaft, auf das Wissen um globale Prozesse sowie auf die Fähigkeit, mit den Auswirkungen dieser Entwicklung - der erhöhten Komplexität - umgehen zu können ${ }^{11}$ (Scheunpflug \& Schröck , 2000, S. 8). Dies bedarf auch einer Qualifizierung für den Umgang mit Ungewissheit, Unsicherheit und Nichtwissen (ebd., S. 16). «Es sind Kenntnisse und Fähigkeiten zu vermitteln, die dazu potentiell beitragen, Schritte auf dem Weg zur Humanisierung dieser Entwicklung zu gehen und nicht nur das Überleben sondern vielleicht auch das gute Leben auf diesem Planeten Erde in der Einen Welt zu ermöglichen» (ebd., S. 9).

Ziel des Globalen Lernens ist es zudem, mit Lernprozessen darauf zu reagieren, dass das menschliche Denken auf unmittelbare «Tat-Folge-Zusam- 
menhänge des konkreten Nahbereichs» (ebd., S. 8) ausgerichtet ist und diese Schwäche zu beheben. "Wenn für Menschen ihre gesellschaftliche Umwelt so komplex und kompliziert geworden ist, dass es nicht mehr möglich ist, Vorhersagen für konkrete Situationen aufzustellen, dann macht es keinen Sinn, sich für eine einzige bestimmte Situation im Leben vorzubereiten, sondern für möglichst viele» (ebd. S. 8). Diese übergeordneten Ziele machen deutlich, dass in diesem Konzept des Globalen Lernens die umfassende Frage, welche Bildung es in einer globalisierten Welt braucht, also die Frage nach einer angemessenen Allgemeinbildung im Vordergrund steht. Dies wird auch aufgrund der Kompetenzbeschreibungen deutlich:

Um die übergeordneten Ziele erreichen zu können, werden gemäss diesem Konzept Kompetenzen aus dem Bereich der Fachkompetenz, der Methodenkompetenz, der Sozialkompetenz sowie der personalen Kompetenz benötigt (ebd., S. 16).

«Unter Fachkompetenzen subsumieren wir die klassischen Kompetenzen des Wissens, des Verstehens und des Urteilens. Es geht darum, Fakten, Regeln, Begriffe zu kennen, Phänomene und Argumente zu durchschauen und Massnahmen oder Zusammenhänge beurteilen zu lernen. (...) Unter Methodenkompetenz fassen wir Fähigkeiten wie Exzerpieren, Nachschlagen, Strukturieren, Planen, Gestalten, Visualisieren oder die selbständige Bearbeitung von komplexen Aufgaben. Soziale Kompetenzen meinen die gerade auch im interkulturellen Zusammenhang so wichtigen Fähigkeiten wie Kooperieren, Zuhören, Begründen, Argumentieren, Fragen, Diskutieren, solidarisches Handeln oder Präsentieren. Wichtige Bestandteile sozialer Kompetenzen im Hinblick auf Globales Lernen sind das Einüben einer differenzierten Sprache und das Erlernen eines sensiblen Umgangs mit unterschiedlichen Sprachen und Sprachfähigkeiten (...). Aufbau einer kommunikativen Kompetenz, Fragehaltung, Umgang mit Nichtverstehen, Offenheit zu Nachfragen sowie Sensibilität für unterschiedliche Körpersprachen sind wichtige Kompetenzen in einer globalisierten Weltgesellschaft und helfen zur Vermeidung von Konflikten. Personale Kompetenzen zeigen sich in Selbstvertrauen, im Aufbau von Werthaltungen, im Üben von Toleranz, in Identifikation und Engagement oder Empathie-Fähigkeiten. Hier geht es auch darum, Widersprüche und Unsicherheiten aushalten zu lernen» (Scheunpflug \& Schröck, 2000, S. 17f.).

\section{Ergebnis des Vergleichs}

Auf den ersten Blick scheinen die verglichenen Konzepte in Bezug auf die Ziele sehr ähnlich. Jedoch unterscheiden sie sich auf den zweiten Blick deutlich. Im BNE-Konzept steht die Beteiligung an der Aus- und Mitgestaltung einer Nachhaltigen Entwicklung im Zentrum - der Umgang mit den gegenwärtigen Gegebenheiten ist dafür eine Voraussetzung, ein Mittel zum Zweck. Im Konzept zum Globalen Lernen ist das übergeordnete Ziel, mit dem Phänomen der Globalisierung und der damit verbundenen Komplexitätssteigerung, also mit 
gegenwärtigen Gegebenheiten, umgehen zu können. Die Befähigung, einen Beitrag zu einem guten Leben in der Einen Welt zu leisten, scheint demgegenüber nachgeordnet bzw. eine Folge davon zu sein.

Im Bereich der Konkretisierung der übergeordneten Ziele finden sich in den Konzepten sehr ähnliche Ziele, vor allem bezogen auf formale Lernziele: z.B. komplexe Phänomene verstehen, mit Ungewissheit umgehen oder argumentieren können. Die Ziele im Konzept zum Globalen Lernen sind vorwiegend als formale Ziele beschrieben - es geht um Fragen der Bildung in einer globalisierten Welt. Demgegenüber umfassen alle Lernziele im Konzept zu BNE sowohl formale als auch materiale Aspekte, und die letztgenannten beziehen sich thematisch immer auf Nachhaltigkeit.

In Bezug auf die Lernziele ist das Bildungsanliegen des Globalen Lernens somit deutlich weiter gefasst als BNE - Nachhaltige Entwicklung stellt in diesem Bildungsanliegen lediglich einen Aspekt der Globalisierung dar, während die Lernziele bei BNE auf Nachhaltigkeit fokussieren und Globalisierung lediglich einen Aspekt darstellt.

Auch wenn in Details Differenzen bestehen, verbinden die Bildungsanliegen die gleichen Grundanliegen

Dieses Argument bezieht sich auf den legitimatorischen Hintergrund der Bildungsanliegen. Damit sind die Phänomene, Anliegen, Diskurse etc. gemeint, die einem Bildungsanliegen, und damit auch einem Konzept zu einem Bildungsanliegen, konstitutiv zu Grunde liegen, die seine Relevanz begründen.

Zum Schluss sollen nun die Legitimationsgrundlagen der verglichenen Bildungsanliegen verglichen werden, und zwar so, wie sie in den hier analysierten didaktischen Konzepten dargelegt werden.

\section{Legitimationsgrundlagen im didaktischen Konzept zu Globalem Lernen}

"Globales Lernen kann als pädagogische Reaktion auf die Entwicklungstatsache zur Weltgesellschaft» (Scheunpflug \& Schröck, 2000, S. 10) und damit auf die Herausforderungen der Globalisierung verstanden werden. Unter dem Titel "Die Probleme» werden Globalisierung und die damit verbundenen Probleme und Veränderungen auf den verschiedenen Ebenen des Zusammenlebens als Ausgangspunkt des Globalen Lernens beschrieben. Dabei wird - jedoch eher nebenbei - erwähnt, dass die Globalisierung auch «neue Chancen und Perspektiven» eröffne (ebd., S. 6). Im analysierten Konzept wird dargelegt, die Krise der Einen Welt sei im Kern eine Lernkrise. Das Denken sowie die Gefühls- und Motivationswelt des Menschen seien nicht auf die globalisierte und komplexe Welt ausgerichtet, sondern noch durch unmittelbare Tat-Folge-Zusammenhänge des konkreten Nahbereichs gekennzeichnet. Diese Schwäche könne durch die menschliche Lern-Fähigkeit ausgeglichen werden. «Deshalb wird auf Lernen soviel Hoffnung gesetzt und Lernen ist deshalb so wichtig geworden wie selten in der Geschichte der Menschheit» (ebd., S. 8). 
Konstitutiv für das Globale Lernen ist also das gesellschaftliche Phänomen der Globalisierung mit den damit einhergehenden Fragen, Problemen und kognitiven Herausforderungen, für die die Kinder und Jugendlichen fit gemacht werden sollen, da dieses gesellschaftliche Phänomen, so die Begründung, den künftigen Lebensalltag stark prägen wird (was früher nicht bzw. weniger der Fall war). Ethische Fragen spielen dabei zwar eine grosse Rolle, sie ergeben sich jedoch aus dem konstitutiven Phänomen und sind selber nicht konstitutiv.

\section{Legitimationsgrundlagen im didaktischen Konzept zur Umweltbildung} «Umweltbildung bezieht sich als Bildungsaufgabe auf die Fähigkeit der jungen Generation zur verantwortungsbewussten Mitgestaltung der Gesellschaft im Hinblick auf die Lösung von Umweltproblemen» (Kyburz-Graber et al., 1997, S. 41). Nachhaltige Entwicklung wird dabei «als Gegenstand sozio-ökologischer Umweltbildung» betrachtet (ebd., S. 28). «Sozio-ökologische Umweltbildung befasst sich also mit anthropogenen Veränderungen in der Natur, die negativ bewertet werden unter Einschluss ihrer anthropogenen Ursachen und der Veränderung dieser Ursachen» (ebd., S. 28).

Konstitutiv für die Umweltbildung sind somit Umweltprobleme, die gelöst oder vermieden werden sollen. Es handelt sich dabei um einen bestimmten Typus gesellschaftlicher Probleme (unangemessener Umgang des Menschen mit der Natur und dessen Folgen). Dahinter steckt der Wunsch, solche Probleme zu lösen, mit den bereits eingetretenen Schäden umzugehen und vergleichbare Probleme in Zukunft zu vermeiden.

Verbunden mit dem Rückgriff auf moderne Umwelt-Theorien und Theorien zum Verhältnis Mensch-Umwelt ist, dass Umweltthemen in einen grösseren Kontext eingebunden werden, insbesondere in den Kontext einer Nachhaltigen Entwicklung. Dieser grössere Kontext ist aber nicht zwingend für die Konzipierung von Umweltbildung.

\section{Ergebnis des Vergleichs}

Für jedes der Bildungsanliegen kristallisiert sich ein anderes Element als konstitutiv heraus:

- Das gesellschaftliche Phänomen der Globalisierung für das Globale Lernen

- Umweltprobleme als bestimmter Typus gesellschaftlicher Probleme für die Umweltbildung

- Die politische Idee der Nachhaltigkeit für BNE

Auch wenn die Wertebasis kompatibel ist und auch, wenn die zugrunde liegenden Ansätze anschlussfähig sind, und auch, wenn im Unterricht auf dieselben Themenfelder Bezug genommen werden kann: Diese konstitutiven Elemente sind nicht identisch. Die drei fächerübergreifenden Bildungsanliegen reagieren auf unterschiedliche politische Diskurse und sind dadurch mit deren unterschiedlichen Grundanliegen verbunden: 
- Globales Lernen reagiert auf den politischen Diskurs über Entwicklungszusammenarbeit und Globalisierung, dessen Anliegen globale Gerechtigkeit ist.

- Umweltbildung reagiert auf den politischen Diskurs über Umweltprobleme, dessen Anliegen die Erhaltung natürlicher Ressourcen und Ökosysteme ist.

- BNE reagiert auf den politischen Diskurs über Nachhaltige Entwicklung, dessen Anliegen das Sichern eines Guten Lebens für alle - inter- und intragenerationell - ist.

\section{Fazit}

Im Hinblick auf die Frage nach dem Verhältnis von BNE und den anderen fächerübergreifenden Bildungsanliegen Umweltbildung und Globales Lernen kann festgestellt werden ${ }^{12}$, dass sich ähnliche oder identische Elemente finden lassen, aber auch Differenzen und sogar Unvereinbarkeiten. Aus der Schnittmenge der didaktischen Strukturelemente ergibt sich jedoch keine Identität oder Ersetzbarkeit dieser Bildungsanliegen. Dies insbesondere dann nicht, wenn die Grundanliegen, auf die die Bildungsanliegen reagieren, so stark differieren, wie dies bei den untersuchten Bildungsanliegen der Fall ist, oder wenn innerhalb der didaktischen Strukturelemente gar Widersprüche bestehen (z.B. Problem- vs. Visionsorientierung). Die Fragen, die de Haan (2002, S. 14) stellt (vgl. Ziffer 1) (z.B. «ist die Beschäftigung mit dem Lebensraum 'Bach', Teil der Umweltbildung oder Teil einer BNE?»), lassen sich so nicht beantworten: Die Beschäftigung mit dem Lebensraum Bach kann sowohl ein Thema in der Umweltbildung als auch in der BNE sein; um diese Frage zu beantworten, müsste die Unterrichtssequenz als Ganzes im Hinblick auf alle didaktischen Strukturelemente in den Blick genommen werden.

Dies bedeutet, dass sich die untersuchten fächerübergreifenden Bildungsanliegen in ihren didaktischen Konzepten nicht verschmelzen lassen, sich nicht gegenseitig ersetzen und auch nicht auseinander hervorgehen, sondern dass es sich vielmehr um je eigenständige Anliegen handelt mit konsistenten didaktischen Konzepten, in denen Inhalte, Prinzipien und Ziele auf differente Legitimationsgrundlagen abgestimmt sind ${ }^{13}$. Das Vorgehen, wie es bei der Entwicklung des Orientierungsrahmens für den Lernbereich «Globale Entwicklung im Rahmen einer Bildung für eine Nachhaltige Entwicklung» (Appelt \& Siege, 2007) unternommen wurde, lässt sich zwar nachvollziehen, jedoch kaum legitimieren. Der Orientierungsrahmen beschreibt für den Bereich «Globale Entwicklung» Kriterien zur Inhaltsauswahl und -gestaltung sowie anzustrebende Kompetenzen, ohne diese in ein Konzept zu BNE einzubinden (was die Verortung dieser Lernziele in umfassendere BNE-Lernziele sowie Ausführungen zu Legitimationsgrundlagen, die mit Nachhaltigkeit kompatibel sein müssten, und Ausführungen zu didaktischen Prinzipien erfordern würde). Würden alle Themen, die für BNE eine Rolle spielen (können), auf diese Weise mit Blick auf 
BNE isoliert behandelt und beschrieben, würde das letztendlich dazu führen, dass für BNE auf ein didaktisches Konzept verzichtet werden müsste. Würde aber auf den Anspruch, der mit einem didaktischen Konzept verbunden ist (theoretische Geschlossenheit etc.), verzichtet, ginge auch die wissenschaftliche Legitimierung des Bildungsanliegens BNE verloren, und damit die Berechtigung, dieses Anliegen in die Curricula integrieren zu wollen.

Aufgrund dieser Ausführungen lässt sich Folgendes festhalten:

$\rightarrow$ Die verschiedenen Bildungsanliegen stehen nicht in Konkurrenz zueinander im Hinblick darauf, welches das bessere Anliegen ist, sondern es sind unterschiedliche Anliegen, die auf andere Herausforderungen und gesellschaftliche Grundanliegen reagieren.

$\rightarrow$ Die verschiedenen Bildungsanliegen sind nicht in jedem Punkt inkommensurabel, sie lassen sich aber nicht gegenseitig substituieren oder ineinander überführen, ohne ihr Wesen zu verlieren.

$\rightarrow$ Für BNE ist ein in sich konsistentes und wissenschaftlich gestütztes didaktisches Konzept erforderlich.

Welches dieser Bildungsanliegen das Sinnvollere oder Relevantere für die Unterrichtspraxis ist, diese Frage kann nicht durch wissenschaftliche Verfahren beantwortet werden, sondern muss auf anderer Ebene entschieden werden. Unter der Annahme, dass die Bildungsanliegen sich von einem gesellschaftlich und bildungstheoretisch berechtigten Anliegen herleiten lassen, gibt es ausserhalb von Wertentscheidungen keine Kriterien, sich für das eine oder das andere eigenständige Bildungsanliegen, zu dem ein theoretisch fundiertes didaktisches Konzept vorliegt, zu entscheiden.

In der Unterrichtspraxis stellt sich nun aber das Problem, dass die für solche Bildungsanliegen zur Verfügung stehende Zeit begrenzt ist und Lehrpersonen und Schulen mit dem Anspruch überfordert sind, allen berechtigten Anliegen (vgl. oben) gerecht zu werden. Wir sehen angesichts des dargelegten Befundes folgende pragmatische Möglichkeiten, mit der Integration von BNE und anderen fächerübergreifenden Bildungsanliegen in Curricula und im Schulalltag umzugehen:

1) Es wird auf bildungspolitischer Ebene entschieden, welche Anliegen in den Curricula aufgenommen und somit als Auftrag an Schule und Lehrpersonen übermittelt werden sollen.

2) In den Curricula werden die verschiedenen Anliegen als gleichwertige Alternativen aufgeführt. Die Entscheidung darüber, welche/s der Anliegen im Schulalltag wirklich umgesetzt wird/werden, wird somit explizit den Lehrpersonen und den Schulen überlassen. 
Beide Varianten bieten Vor- und Nachteile. Bei der ersten Variante stellt sich die heikle Frage der Begründbarkeit der Vorgaben: es sollten klare Kriterien vorliegen, die den Entscheid legitimieren. Dass solche Kriterien aufgestellt werden können, die auf Akzeptanz stossen, stellt hier eine Schwierigkeit dar. Der Vorteil dieser Variante bestünde darin, dass ein kontinuierlicher Aufbau des Unterrichts über die verschiedenen Schulstufen hinweg nach einem Kompetenzmodell erfolgen könnte.

Bei der zweiten Variante droht die Gefahr, dass Lehrpersonen sich für die Vermittlung verschiedenster fächerübergreifender Bildungsanliegen entscheiden und somit die einzelnen Anliegen nur oberflächlich aufgegriffen werden. Auch ist es nicht möglich, über die Schulstufen hinweg ein Kompetenzmodell zugrunde zu legen. Der Vorteil dieser Variante bestünde darin, dass Lehrpersonen flexibel auf aktuelle Herausforderungen reagieren könnten und eine Profilierung von Lehrpersonen und Schulen möglich würde. ${ }^{14}$

\section{Anmerkungen}

1 In diesem Diskurs wird BNE in der Regel auf eine der Funktionen von Bildung im Kontext Nachhaltiger Entwicklung (Di Giulio \& Künzli, 2006) bezogen, nämlich auf die Funktion der Vermittlung spezifischer Kompetenzen für eine Nachhaltige Entwicklung. Dieses Verständnis von BNE liegt auch den nachfolgenden Ausführungen zugrunde.

2 In Bezug auf die Legitimationsgrundlagen der fächerübergreifenden Bildungsanliegen zeigt sich der Bezug zu bildungspolitischen, gesellschaftlichen Diskursen.

3 Die Auswahl wurde im Wissen darum getroffen, dass die entsprechenden Bildungsanliegen derzeit intensiv diskutiert werden und Veränderungen unterliegen. Das bedeutet auch, dass ein solcher Vergleich immer nur eine Momentaufnahme darstellen kann.

4 Es wurden deutschsprachige Konzepte ausgewählt, um von vergleichbaren Schulsystemen ausgehen zu können.

5 Umso weniger kann der Anspruch auf eine vollständige Abdeckung der analysierten Bildungsanliegen erhoben werden. Es wurden weiter keine Konzepte analysiert, die darlegen, wie die entsprechende Thematik (z.B. Globale Entwicklung) in BNE integriert werden könnte (wie dies z.B. im Orientierungsrahmen für den Lernbereich Globale Entwicklung im Rahmen einer Bildung für nachhaltige Entwicklung (Appelt \& Siege, 2007) der Fall ist).

6 Das didaktische Konzept der sozio-ökologischen Umweltbildung von Kyburz-Graber et al. ist ausführlich in der Publikation von 1997 ausgeführt. Es liegt einem Forschungsprojekt zugrunde und wurde im Hinblick darauf leicht adaptiert. Die Veränderungen wurden in der Publikation von 2000 dargelegt. Für den Vergleich wurden beide Publikationen einbezogen.

7 Dieses Konzept wurde im Rahmen des Projekts «Bildung für eine nachhaltige Entwicklung: Didaktische Konzeption und Umsetzung in die Schulpraxis» (SNF Nr. 1163780.00) entwickelt. Das Projekt wurde an der Interfakultären Koordinationsstelle für Allgemeine Ökologie (IKAÖ) der Universität Bern in Kooperation mit dem Institut für Erziehungswissenschaft der Universität Bern durchgeführt.

8 Ein vergleichbares übergeordnetes Ziel wird im Konzept von de Haan mit dem Begriff «Gestaltungskompetenz» bezeichnet (de Haan, 2008).

9 Die Richtziele werden nach dem Vorschlag der OECD (DEELSA, 2002) nach den drei Kategorien selbstständig handeln (acting autonomously), Medien und Tools interaktiv verwenden (using tools interactively) und in sozial heterogenen Gruppen tätig sein 
(functioning in socially heterogeneous groups) geordnet. Für eine weitere Ausdifferenzierung der Richtziele in Teilkompetenzen vgl. Bertschy et al. (2007).

10 Das didaktische Konzept der sozio-ökologischen Umweltbildung von Kyburz-Graber et al. $(1997,2000)$ bezieht sich explizit auf die Idee der Nachhaltigkeit. Es wird jedoch ebenso explizit der Begriff (sozio-ökologische) Umweltbildung beibehalten, u.a. um sich abzugrenzen von Nachhaltigkeit als politisches Programm und als gesellschaftliches Leitbild.

11 Die Entwicklung zu einer Weltgesellschaft kann unter verschiedenen Blickwinkeln bearbeitet werden (Seite der Menschenrechte, Weltwirtschaft, globale Umweltgefährdungen oder Nachhaltigkeit). Die Idee der Nachhaltigen Entwicklung bildet keine eigentliche Grundlage des Konzepts, es ist «eine Perspektive unter anderen».

12 Die nachfolgenden Ausführungen beziehen sich zum einen auf die verglichenen didaktischen Konzepte und zum anderen auf das vorgängig dargelegte Verständnis von BNE. Ob sich die Ergebnisse auch auf den Vergleich mit anderen didaktischen Konzepten übertragen lassen, müsste Gegenstand weiterer Forschungsarbeiten sein.

13 Damit ist jedoch nicht die Handhabung auf Ebene der konkreten Unterrichtspraxis angesprochen. Es ist möglich, dass Lehrerinnen und Lehrer Elemente aus den drei untersuchten Bildungsanliegen in einer einzigen Unterrichtsreihe umsetzen.

14 Voraussetzung dieser Variante ist jedoch die Ausbildung der Lehrpersonen in den verschiedenen fächerübergreifenden Bildungsanliegen. Das Problem der Überfrachtung und Überforderung wird somit jedoch an Lehrerinnen- und Lehrerbildungsinstitutionen weitergegeben.

\section{Literatur}

Apel, H. J. (1995). Theorie der Schule. Donauwörth: Ludwig Auer.

Appelt, D. \& Siege, H. (2007). Orientierungsrahmen für den Lernbereich Globale Entwicklung im Rahmen einer Bildung für Nachhaltige Entwicklung. Zugriff am 15.10.2009 unter http://www.globaleslernen.de/coremedia/generator/ewik/de/Downloads/Dokumente/Ori entierungsrahmen_20f_C3_BCr_20Globales_20Lernen.pdf

Bertschy, F., Gingins, F., Künzli, Ch., Di Giulio, A. \& Kaufmann-Hayoz, R. (2007). Bildung für eine Nachhaltige Entwicklung in der Grundschule. Schlussbericht zum Expertenmandat der EDK: "Nachhaltige Entwicklung in der Grundschulausbildung - Begriffsklärung und Adaption». Zugriff am 15.10.2009 unter http://www.Hedudoc.ch/record/24373/files/BNE_Schlussbericht_2007_d.pdf

Bolscho, D., Hauenschild, K. \& Rode, H. (2008). Bildung für eine nachhaltige Entwicklung in der Grundschule. Ausgewählte Ergebnisse einer Pilotstudie mit Lehrerinnen und Lehrern. In H. Giest \& J. Wiesemann (Hrsg.), Kind und Wissenschaft. Welches Wissenschaftsverständnis hat der Sachunterricht? (S. 301-312). Bad Heilbrunn: Julius Klinkhardt.

DEELSA (2002). Definition and selection of competences (deseco): Theoretical and conceptual foundations. Strategy paper. Organisation for economic co-operation and development. Zugriff am 15.10.2009 unter

http://www.deseco.admin.ch/bfs/deseco/en/index/02.parsys.34116.downloadList.87902. DownloadFile.tmp/oecddesecostrategypaperdeelsaedcericd20029.pdf

De Haan, G. (2001). Was meint «Bildung für nachhaltige Entwicklung» und was können eine globale Perspektive und neue Kommunikationsmöglichkeiten zur Weiterentwicklung beitragen? In O. Herz, H. Seybold \& G. Strobl (Hrsg.), Bildung für eine nachhaltige Entwicklung. Globale Perspektiven und neue Kommunikationsmedien (S. 29-45). Opladen: Leske und Budrich.

De Haan, G. (2002). Die Kernthemen der Bildung für eine nachhaltige Entwicklung. ZEP Zeitschrift für internationale Bildungsforschung und Entwicklungspädagogik, 1, 13-20. 
De Haan, G. (2008). Gestaltungskompetenz als Kompetenzkonzept der Bildung für nachhaltige Entwicklung. In I. Bormann \& G. de Haan (Hrsg.), Kompetenzen der Bildung für nachhaltige Entwicklung (S. 23-43). Wiesbaden: VS Verlag für Sozialwissenschaften.

Di Giulio, A. (2004). Die Idee der Nachhaltigkeit im Verständnis der Vereinten Nationen. Anspruch, Bedeutung, Schwierigkeiten. Münster: LIT.

Di Giulio, A. \& Künzli, Ch. (2006). Partizipation von Kindern und Jugendlichen im Kontext von Bildung und nachhaltiger Entwicklung. In C. Quesel \& F. Oser (Hrsg.), Die Mühen der Freiheit. Probleme und Chancen der Partizipation von Kindern und Jugendlichen (S. 205219). Chur: Rüegger.

Durdel, A. (2002). Der Bildungsbegriff als Konstruktion. Orientierungs- und handlungsleitendes Potenzial des Bildungsbegriffes. Hamburg: Dr. Kovac.

Einsiedler, W. (1994). Sollen Lehrer Sozialpädagogen werden? Bayerische Schule, 12, 25-28.

Fien, J. (2001). Education for Sustainability: Reorientating Australian schools for a sustainable future. Tela: Australian Conservation Foundation, Griffith University.

Hauff, V. (Hrsg.). (1987). Unsere gemeinsame Zukunft. Brundtland-Bericht der Weltkommission für Umwelt und Entwicklung. Greven: Eggenkamp.

Helbling, R.\& Schwarz, V. (2005). «Bildung für eine nachhaltige Entwicklung soll curricular werden». Zum Stand der Diskussion in der Schweiz. ZEP - Zeitschrift für internationale Bildungsforschung und Entwicklungspädagogik, 3, 27-32.

Herzog, W. \& Künzli David, Ch. (2007). Nachhaltigkeit in der Erziehungswissenschaft. Schlaglichter auf einen unabgeschlossenen Diskurs. In SAGW (Hrsg.), Nachhaltigkeitsforschung - Perspektiven der Sozial- und Geisteswissenschaften (S. 281-304). Bern: SAGW.

Hoppe, H. (1996). Subjektorientierte politische Bildung. Begründung einer Biografiezentrierten Didaktik der Gesellschaftswissenschaften. Opladen: Leske und Budrich.

Kübler, D., Kissling-Näf, I. \& Zimmermann, W. (2001). Wie nachhaltig ist die Schweizer Forstpolitik? Ein Beitrag zur Kriterien- und Indikatorendiskussion. Basel: Helbling \& Lichtenhahn.

Künzli David, Ch. (2007). Zukunft mitgestalten. Bildung für eine nachhaltige Entwicklung Didaktisches Konzept und Umsetzung in der Grundschule. Bern: Haupt.

Künzli David, Ch. \& Bertschy, F. (2008). Didaktisches Konzept Bildung für eine nachhaltige Entwicklung. (3. überarbeitete Auflage). Zugriff am 15.09.2009 unter http://www.ikaoe.unibe.ch/forschung/bineu/Did.Konzept_3.Fassung_Feb08.pdf

Kyburz-Graber, R., Högger, D. \& Wyrsch, A. (2000). Sozio-ökologische Umweltbildung in der Praxis: Hindernisse, Bedingungen, Potentiale. Schlussbericht zum Forschungsprojekt "Bildung für eine nachhaltige Schweiz» SPP-Umwelt des Schweizerischen Nationalfonds. Universität Zürich.

Kyburz-Graber, R., Rigendinger, L., Hirsch Hadorn, G. \& Werner Zentner, K. (1997). Sozioökologische Umweltbildung. Hamburg: Krämer.

Mayring, Ph. (2000). Qualitative Inhaltsanalyse. Grundlagen und Techniken. Weinheim: Beltz.

Minsch, J., Eberle, A., Meier, B. \& Schneidewind, U. (1996). Mut zum ökologischen Umbau. Innovationsstrategien für Unternehmen, Politik und Akteurnetze. Basel: Birkhäuser.

Nagel, U., Kern, W. \& Schwarz, V. (2006). Beiträge zur Festlegung von Kompetenzen und Standards für die Bildung für Nachhaltige Entwicklung - unter den Aspekten Umweltbildung, Gesundheitsbildung und Globales Lernen. Schlussbericht. Pädagogische Hochschule Zürich.

Rost, J. (2002). Umweltbildung - Bildung für eine nachhaltige Entwicklung: Was macht den Unterschied? ZEP - Zeitschrift für internationale Bildungsforschung und Entwicklungspädagogik, 1, 7-12.

Scheunpflug, A. (2003). Stichwort Globalisierung und Erziehungswissenschaft. Zeitschrift für Erziehungswissenschaft, 6 (2), 159-172. 
Scheunpflug, A. (2007). Die konzeptionelle Weiterentwicklung des Globalen Lernens. Die Debatten der letzten zehn Jahre. In VENRO (Hrsg.), Jahrbuch Globales Lernen 2007/2008 (S. 11-21). Bonn: Venro.

Scheunpflug, A. \& Schröck, N. (2000). Globales Lernen. Einführung in eine pädagogische Konzeption zur entwicklungsbezogenen Bildung. Stuttgart: Brot für die Welt.

Schorch, G. (2007). Studienbuch Grundschulpädagogik. Bad Heilbrunn: Klinkhardt.

Schlagworte: Bildungsanliegen, Bildung für eine Nachhaltige Entwicklung, Globales Lernen, Umweltbildung, didaktische Konzepte

\section{Éducation au développement durable, éducation dans une perspective globale et éducation à l’environnement: une comparaison}

\section{Résumé}

De nombreuses initiatives visent aujourd'hui à ancrer l'éducation au développement durable (EDD) dans le système éducatif. Dans ce contexte, une question de fond porte sur les relations entre l'EDD, l'éducation à l'environnement et l'éducation dans une perspective globale. Certains acteurs craignent que l'EDD ne fasse rien d'autre qu'alourdir le catalogue déjà étoffé des "éducations à...». On peut cependant relever que bien des "éducations à..." ont des liens avec l'EDD dont elles abordent certains aspects spécifiques. C'est en particulier le cas de l'éducation dans une perspective globale et de l'éducation à l'environnement. A ce titre, l'EDD pourrait être vue comme un prolongement d'autres intentions de formation générale.

Le présent article développe cette question des relations entre l'EDD, l'éducation dans une perspective globale et l'éducation à l'environnement.

Mots clés: Intentions de formation, éducation en vue du développement durable, éducation dans une perspective globale, éducation à l'environnement, concepts didactiques 


\section{L'educazione per uno sviluppo sostenibile a confronto con un'educazione di prospettiva globale e un'educazione ecologica}

\section{Riassunto}

Numerose sono oggi le iniziative tese ad ancorare l'educazione per uno sviluppo sostenibile (ESS) nel sistema educativo. In questo contesto, una delle questioni cruciali riguarda il rapporto tra ESS e educazione aperta alla prospettiva globale e educazione ambientale. Diversi autori temono che la ESS non faccia che allungare il già lungo elenco delle "educazione per...". D'altro canto si può costatare come contenuti formativi a carattere trasversale, in particolare attinenti all'educazione ambientale e all'educazione in prospettiva globale, facciano riferimento alla ESS, fornendo a quest'ultima una sorta di legittimazione. L'articolo entra nel merito di questi interrogativi in relazione alla ESS, all'educazione in prospettiva globale e all'educazione all'ambiente.

Parole chiave: Obiettivi formativi, Educazione allo sviluppo sostenibile, educazione ad aspirazione globale, educazione ambientale, concetti didattici.

\section{Education for a sustainable development in comparison with global education and environmental education}

\footnotetext{
Abstract

At present, there are several initiatives taking place with the goal to anchor education for a sustainable development (ESD) within the educational system. Within this context, a pivotal question arises regarding the relationship between ESD, global education and environmental education. On one hand, it is feared that ESD adds yet another educational sub-area to the already vast catalogue, on the other hand, observations suggest that demands for interdisciplinary educational requirements, particularly global education and environmental education, are often related to ESD - thus, ESD apparently can be connected to existing interdisciplinary demands for education.

This article is dedicated to this question concerning the relationship between ESD and global education as well as environmental education.

Key words: Education for a sustainable development, environmental education, global education, interdisciplinary educational requirements, pedagogical concepts
} 